\title{
A dose-independent association of triglyceride levels with all-cause mortality among adults population
}

\author{
Yu-qing Huang ${ }^{1 \dagger}$, Xiao-cong Liu ${ }^{1 \dagger}$, Kenneth Lo ${ }^{1,2,3}$, Ying-qing Feng ${ }^{1 *}$ and Bin Zhang ${ }^{1 *}$
}

\begin{abstract}
Background: The relationship between triglyceride (TG) level and the mortality risk of all-cause and cardiovascular disease is not entirely consistent among adults.

Methods: The present analysis included adult participants from National Health and Nutrition Examination Surveys (NHANES) between the periods 1999-2014. The levels of TG were categorized into $<150,150-199,200-250$ and $\geq$ $250 \mathrm{mg} / \mathrm{dL}$ respectively. Multivariate Cox regression analysis, stratified analysis and generalized additive model were conducted to reveal the correlation between TG and mortality risk. Results were presented in hazard ratio (HRs) and 95\% confidence intervals (Cls).

Results: There were 18,781 (9130 males, mean age was 45.64 years) participants being included in the analysis. The average follow-up period was 8.25 years, where 1992 (10.61\%) cases of all-cause and 421 (2.24\%) cardiovascular death have occurred. In the multivariate Cox model, every $1 \mathrm{mg} / \mathrm{dL}$ raise in TG has significantly associated with allcause mortality (HR: 1.08, 95\% Cl: 1.02, 1.15) but not cardiovascular mortality (HR: 1.10, 95\% Cl: 0.97, 1.24). When using $\mathrm{TG}<150 \mathrm{mg} / \mathrm{dL}$ as reference, $\mathrm{TG} \geq 250 \mathrm{mg} / \mathrm{dL}$ associated with death from all-cause $(\mathrm{HR}=1.34,95 \% \mathrm{Cl}: 1.12$, $1.60 ; P=0.0016$ but not cardiovascular death $(\mathrm{HR}=1.26,95 \% \mathrm{Cl}: 0.85,1.88 ; P=0.2517)$. According to smoothing spline plots, the risk of all-cause was the lowest when TG was approximately $135 \mathrm{mg} / \mathrm{dL}$.
\end{abstract}

Conclusion: TG might have a dose-independent association with all-cause mortality among adults in United States.

Keywords: Triglyceride, All-cause mortality, Cardiovascular mortality, Adult population, Nonlinear, Dose-independent

\section{Introduction}

Multiple epidemiological and clinical studies have reported the linkage between elevated triglyceride (TG) concentrations and cardiovascular diseases (CVD) [1-5]. The Bezafibrate Infarction Prevention Registry study revealed that elevated TG associated with higher mortality risk in patients with coronary heart disease [6]. More

\footnotetext{
*Correspondence: 651792209@qq.com; 3418989350@qq.com

†Yu-qing Huang and Xiao-cong Liu contributed equally to this work.

'Department of Cardiology, Guangdong Cardiovascular Institute, Guangdong Provincial People's Hospital, Guangdong Academy of Medical Sciences,

South China University of Technology School of Medicine, No. 106,

Zhongshan Second Road, Yuexiu District, Guangzhou 510080, China

Full list of author information is available at the end of the article
}

recently, a meta-analysis of 61 cohorts demonstrated relationship between TG levels and CVD in a doseresponse manner [7]. Another meta-regression analysis of 49 randomized trials has found that lowering TG could reduce the risk of major vascular events, which was independent from the levels of circulating low density lipoprotein cholesterol (LDL-C) [8]. However, the relation between TG and mortality was often attenuated after being adjusting for cardiovascular risk factors. Currently, whether triglyceride concentration was independently related to all-cause or cause-specific mortality has been controversial. Davis, et al. [9] suggested that the levels of TG had no independent association with

(c) The Author(s). 2020 Open Access This article is licensed under a Creative Commons Attribution 4.0 International License, which permits use, sharing, adaptation, distribution and reproduction in any medium or format, as long as you give appropriate credit to the original author(s) and the source, provide a link to the Creative Commons licence, and indicate if changes were made. The images or other third party material in this article are included in the article's Creative Commons licence, unless indicated otherwise in a credit line to the material. If material is not included in the article's Creative Commons licence and your intended use is not permitted by statutory regulation or exceeds the permitted use, you will need to obtain permission directly from the copyright holder. To view a copy of this licence, visit http://creativecommons.org/licenses/by/4.0/ The Creative Commons Public Domain Dedication waiver (http://creativecommons.org/publicdomain/zero/1.0/) applies to the data made available in this article, unless otherwise stated in a credit line to the data. 
mortality due to coronary disease. Khawaja et al. [10] demonstrated that lower TG $(\mathrm{TG}<200 \mathrm{mg} / \mathrm{dL})$ at admission was associated with a higher risk of mortality in participants with myocardial infarction. Considering the discrepancies in findings, the prospective relationship between serum TG levels and mortality was explored among adults in United States (US).

\section{Methods}

\section{Subjects enrollment}

The data source was from National Health and Nutrition Examination Surveys (NHANES) between the periods 1999-2014. NHANES was a nationwide study conducted by Centers for Disease Control and Prevention (CDC) in United States. Serum TG was determined among NHANES participants aged $\geq 18$ years old. After excluding participants younger than 18 , people with missing serum TG levels or with cancer at baseline, 18, 781 participants were included (Fig. 1). The Institutional Review Board of the CDC has approved the protocol of survey (Protocol 98-12, 2005-06 and 2011-17). Participants provided informed consent in written form before the start of the study.

\section{Assessment of exposure}

Standardized procedures and methods were used for collecting serum samples. Serum samples were collected from venous vessels in the morning. Serum circulating TG and total cholesterol (TC) levels were measured enzymatically, while direct immunoassay or precipitation was used to determine the level of highdensity lipoprotein cholesterol (HDL-C) [11]. Hitachi 704 Analyzer (Boehringer Mannheim Diagnostics, Indianapolis, IN) was used to measure serum TC, HDL$\mathrm{C}$ and TG [12]. If TG was $\leq 400 \mathrm{mg} / \mathrm{dL}$, Friedewald formula was adapted to calculate the value of LDL-C [13].

\section{Assessment of covariates}

At baseline, socio-demographics and lifestyle factors (including age, race/ethnicity, gender, smoking status, diet habits and education levels), data on physical examination (height, weight, blood pressure, estimated glomerular filtration rate (eGFR)), disease history and medication history were acquired. Hypertension was defined by self-reported history, systolic/diastolic blood pressure $(\mathrm{SBP} / \mathrm{DBP}) \geq 140 / 90 \mathrm{mmHg}$, or taking drugs to reduce blood pressure [14]. Diabetes was defined by selfreported history, taking hypoglycemic medications, fasting serum glucose level $\geq 7.0 \mathrm{mmol} / \mathrm{L}$ or hemoglobin $\mathrm{A} 1 \mathrm{C} \geq 6.5 \%[15]$.

\section{Assessment of outcome}

The outcomes of this study were death from all-cause and CVD. Mortality status was ascertained from NHAN ES until death or 31st December 2015, whichever occurred first. Codes in the 10th edition of the International Classification of Diseases including I00-I09, I20I51, I11 and I13 were used to derive the cause of CVD death [16].

\section{Statistical analyses}

Descriptive statistics was presented according to the levels of TG $(<150,150-200,200-249, \geq 250 \mathrm{mg} / \mathrm{dL})$ at baseline. Differences by TG levels were explored by one-way analysis of variance, Kruscal Whallis $H$ test and chi-square tests. Multivariate Cox regression models with hazard ratios (HRs) and 95\% confidence intervals (CIs) were built to assess the death from allcause and CVD. Model I was crude model and no

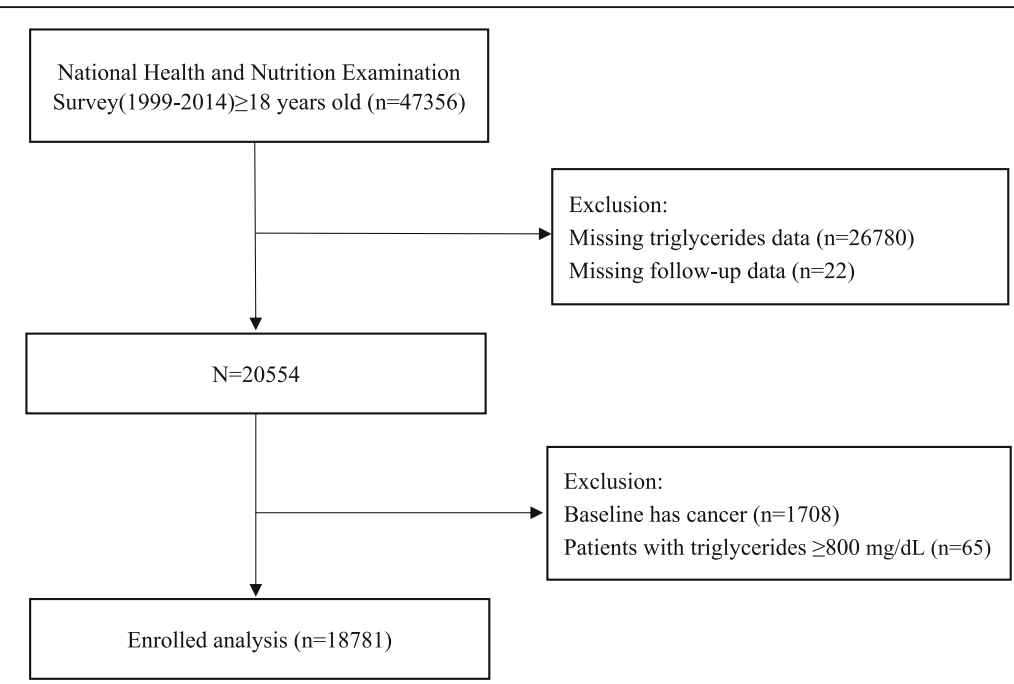

Fig. 1 Research flow chart 
Table 1 Demographic and clinical characteristics according to triglyceride levels

\begin{tabular}{|c|c|c|c|c|c|c|}
\hline \multirow[t]{2}{*}{ Characteristic } & \multirow[b]{2}{*}{ Total } & \multicolumn{4}{|c|}{ Triglycerides, mg/dL } & \multirow[t]{2}{*}{$P$-value } \\
\hline & & $<150$ & $150-199$ & $200-250$ & $\geq 250$ & \\
\hline Number & 18,781 & 13,350 & 2576 & 1322 & 1533 & \\
\hline Age, years & $45.64 \pm 18.94$ & $44.30 \pm 19.15$ & $49.09 \pm 18.45$ & $49.63 \pm 18.23$ & $48.12 \pm 16.92$ & $<0.001$ \\
\hline Gender, n (\%) & & & & & & $<0.001$ \\
\hline Male & $9130(48.61)$ & $6246(46.79)$ & $1292(50.16)$ & $726(54.92)$ & $866(56.49)$ & \\
\hline Female & $9651(51.39)$ & $7104(53.21)$ & $1284(49.84)$ & $596(45.08)$ & $667(43.51)$ & \\
\hline Race, n (\%) & & & & & & $<0.001$ \\
\hline Non-white & $10,628(56.59)$ & $7806(58.47)$ & $1367(53.07)$ & $675(51.06)$ & $780(50.88)$ & \\
\hline White & $8153(43.41)$ & $5544(41.53)$ & $1209(46.93)$ & 647 (48.94) & $753(49.12)$ & \\
\hline Smoking, n (\%) & & & & & & $<0.001$ \\
\hline No & $9463(54.54)$ & $6953(57.31)$ & $1201(48.86)$ & 619 (48.59) & $690(46.43)$ & \\
\hline Yes & $7887(45.46)$ & $5179(42.69)$ & $1257(51.14)$ & $655(51.41)$ & $796(53.57)$ & \\
\hline Education level, n (\%) & & & & & & $<0.001$ \\
\hline Less than high school & $5010(29.15)$ & $3226(26.90)$ & $829(33.93)$ & $437(34.46)$ & $518(34.91)$ & \\
\hline High school or above & $12,177(70.85)$ & $8766(73.10)$ & $1614(66.07)$ & $831(65.54)$ & $966(65.09)$ & \\
\hline Body mass index, $\mathrm{kg} / \mathrm{m}^{2}$ & $28.46 \pm 6.68$ & $27.75 \pm 6.78$ & $29.94 \pm 6.26$ & $30.29 \pm 6.08$ & $30.58 \pm 5.78$ & $<0.001$ \\
\hline Systolic blood pressure, $\mathrm{mmHg}$ & $122.53 \pm 19.03$ & $121.15 \pm 18.72$ & $124.98 \pm 19.31$ & $126.52 \pm 19.49$ & $126.96 \pm 19.23$ & $<0.001$ \\
\hline Diastolic blood pressure, $\mathrm{mmHg}$ & $68.88 \pm 13.25$ & $68.22 \pm 12.88$ & $70.26 \pm 13.41$ & $70.28 \pm 14.15$ & $71.12 \pm 14.73$ & $<0.001$ \\
\hline Energy, kcal & $2149.28 \pm 1020.81$ & $2146.82 \pm 1032.72$ & $2112.49 \pm 991.50$ & $2144.78 \pm 957.29$ & $2237.06 \pm 1015.15$ & 0.003 \\
\hline $\mathrm{eGFR}, \mathrm{mg} / \mathrm{min} / 1.73 \mathrm{~m}^{2}$ & $92.49 \pm 29.80$ & $93.07 \pm 28.43$ & $90.16 \pm 31.21$ & $91.43 \pm 34.66$ & $92.23 \pm 34.06$ & $<0.001$ \\
\hline C-reactive protein, mg/L & $0.45 \pm 0.86$ & $0.42 \pm 0.87$ & $0.51 \pm 0.97$ & $0.51 \pm 0.66$ & $0.53 \pm 0.72$ & $<0.001$ \\
\hline \multicolumn{7}{|l|}{ Triglycerides } \\
\hline $\mathrm{mg} / \mathrm{dL}$ & $131.38 \pm 87.24$ & $89.44 \pm 30.20$ & $171.66 \pm 14.46$ & $222.04 \pm 14.32$ & $350.73 \pm 107.34$ & $<0.001$ \\
\hline $\mathrm{mmol} / \mathrm{L}$ & $1.48 \pm 0.98$ & $1.01 \pm 0.34$ & $1.94 \pm 0.16$ & $2.51 \pm 0.16$ & $3.96 \pm 1.21$ & $<0.001$ \\
\hline \multicolumn{7}{|l|}{ Low density lipoprotein cholesterol } \\
\hline $\mathrm{mg} / \mathrm{dL}$ & $114.90 \pm 35.92$ & $111.57 \pm 33.69$ & $125.44 \pm 37.33$ & $125.36 \pm 40.70$ & $118.22 \pm 44.07$ & $<0.001$ \\
\hline $\mathrm{mmol} / \mathrm{L}$ & $2.97 \pm 0.93$ & $2.89 \pm 0.87$ & $3.24 \pm 0.97$ & $3.24 \pm 1.05$ & $3.06 \pm 1.14$ & $<0.001$ \\
\hline \multicolumn{7}{|l|}{ Total cholesterol } \\
\hline $\mathrm{mg} / \mathrm{dL}$ & $194.24 \pm 42.18$ & $185.88 \pm 38.09$ & $207.80 \pm 40.78$ & $215.37 \pm 44.77$ & $226.00 \pm 48.30$ & $<0.001$ \\
\hline $\mathrm{mmol} / \mathrm{L}$ & $5.02 \pm 1.09$ & $4.81 \pm 0.99$ & $5.37 \pm 1.05$ & $5.57 \pm 1.16$ & $5.84 \pm 1.25$ & $<0.001$ \\
\hline \multicolumn{7}{|l|}{ High density lipoprotein cholesterol } \\
\hline $\mathrm{mg} / \mathrm{dL}$ & $53.33 \pm 15.65$ & $56.43 \pm 15.46$ & $48.01 \pm 13.17$ & $45.95 \pm 13.05$ & $41.73 \pm 12.96$ & $<0.001$ \\
\hline $\mathrm{mmol} / \mathrm{L}$ & $1.38 \pm 0.40$ & $1.46 \pm 0.40$ & $1.24 \pm 0.34$ & $1.19 \pm 0.34$ & $1.08 \pm 0.34$ & $<0.001$ \\
\hline Diabetes, n (\%) & & & & & & $<0.001$ \\
\hline No & $15,898(84.68)$ & $11,739(87.95)$ & $2066(80.26)$ & $1006(76.15)$ & $1087(70.91)$ & \\
\hline Yes & $2877(15.32)$ & $1608(12.05)$ & $508(19.74)$ & $315(23.85)$ & $446(29.09)$ & \\
\hline Hypertension, n (\%) & & & & & & $<0.001$ \\
\hline No & $11,387(60.70)$ & $8587(64.38)$ & $1384(53.83)$ & $678(51.32)$ & $738(48.20)$ & \\
\hline Yes & $7374(39.30)$ & $4751(35.62)$ & $1187(46.17)$ & $643(48.68)$ & $793(51.80)$ & \\
\hline Cardiovascular disease, n (\%) & & & & & & $<0.001$ \\
\hline No & $15,712(91.26)$ & $11,076(92.22)$ & $2197(89.64)$ & $1118(88.03)$ & $1321(88.96)$ & \\
\hline Yes & $1504(8.74)$ & $934(7.78)$ & $254(10.36)$ & $152(11.97)$ & $164(11.04)$ & \\
\hline Antihypertensive drugs, n (\%) & & & & & & $<0.001$ \\
\hline
\end{tabular}


Table 1 Demographic and clinical characteristics according to triglyceride levels (Continued)

\begin{tabular}{|c|c|c|c|c|c|c|}
\hline \multirow[t]{2}{*}{ Characteristic } & \multirow[b]{2}{*}{ Total } & \multicolumn{4}{|c|}{ Triglycerides, mg/dL } & \multirow[t]{2}{*}{$P$-value } \\
\hline & & $<150$ & $150-199$ & $200-250$ & $\geq 250$ & \\
\hline Yes & $4423(23.55)$ & $2793(20.92)$ & $741(28.77)$ & 409 (30.94) & $480(31.31)$ & \\
\hline Hypoglycemic agents, n (\%) & & & & & & $<0.001$ \\
\hline No & $17,309(92.16)$ & $12,516(93.75)$ & $2328(90.37)$ & $1167(88.28)$ & $1298(84.67)$ & \\
\hline Yes & $1472(7.84)$ & $834(6.25)$ & $248(9.63)$ & 155 (11.72) & $235(15.33)$ & \\
\hline Lipid-lowering drugs, n (\%) & & & & & & $<0.001$ \\
\hline No & $16,560(88.17)$ & $11,956(89.56)$ & $2193(85.13)$ & $1113(84.19)$ & $1298(84.67)$ & \\
\hline Yes & $2221(11.83)$ & $1394(10.44)$ & $383(14.87)$ & $209(15.81)$ & 235 (15.33) & \\
\hline Antiplatelet drugs, n (\%) & & & & & & $<0.001$ \\
\hline No & $18,460(98.29)$ & $13,153(98.52)$ & $2508(97.36)$ & $1292(97.73)$ & $1507(98.30)$ & \\
\hline Yes & $321(1.71)$ & $197(1.48)$ & $68(2.64)$ & $30(2.27)$ & $26(1.70)$ & \\
\hline Cardiovascular mortality, n (\%) & & & & & & $<0.001$ \\
\hline No & $18,360(97.76)$ & $13,108(98.19)$ & $2493(96.78)$ & $1275(96.44)$ & $1484(96.80)$ & \\
\hline Yes & $421(2.24)$ & $242(1.81)$ & $83(3.22)$ & $47(3.56)$ & $49(3.20)$ & \\
\hline All-cause mortality, n (\%) & & & & & & $<0.001$ \\
\hline No & 16,789 (89.39) & $12,114(90.74)$ & $2243(87.07)$ & $1131(85.55)$ & $1301(84.87)$ & \\
\hline Yes & $1992(10.61)$ & $1236(9.26)$ & $333(12.93)$ & $191(14.45)$ & $232(15.13)$ & \\
\hline
\end{tabular}

Note: Subgroup differences were tested by one-way analysis of variance for continuous variables and by chi-square for categorical variables Values are mean \pm standardized differences or number (\%)

$n$ number; eGFR estimated glomerular filtration rate

confounders were included. In Model II, age, sex and body mass index (BMI) were adjusted. In Model III, race, level of education, smoking status, SBP, energy intake, C-reactive protein, eGFR, TC, HDL-C, disease history and the use of medication were additionally adjusted. Differences in survival rates by TG levels were analyzed by Kaplan-Meier curves. Restricted cubic spline analysis was used to reveal how serum TG might relate to all-cause and CVD mortality. A generalized additive model was used to assess any nonlinear relationship. If a nonlinear relationship was detected, Cox proportional hazards models were built on both sides of the inflection point. Results from Cox regression were stratified by age at baseline $(<65$ or $\geq 65$ years), sex (man or woman), ethnicity (White or non-White), BMI categories $\left(<25\right.$ or $\left.\geq 25 \mathrm{~kg} / \mathrm{m}^{2}\right)$, history of diabetes or hypertension, history of CVD and the use of lipid-lowering drugs (all categorized into yes or no). Statistical analyses were performed by $\mathrm{R}$ software with the version of 3.3.2 (Vienna, Austria) and $P<0.05$ was regarded as statistically significant.
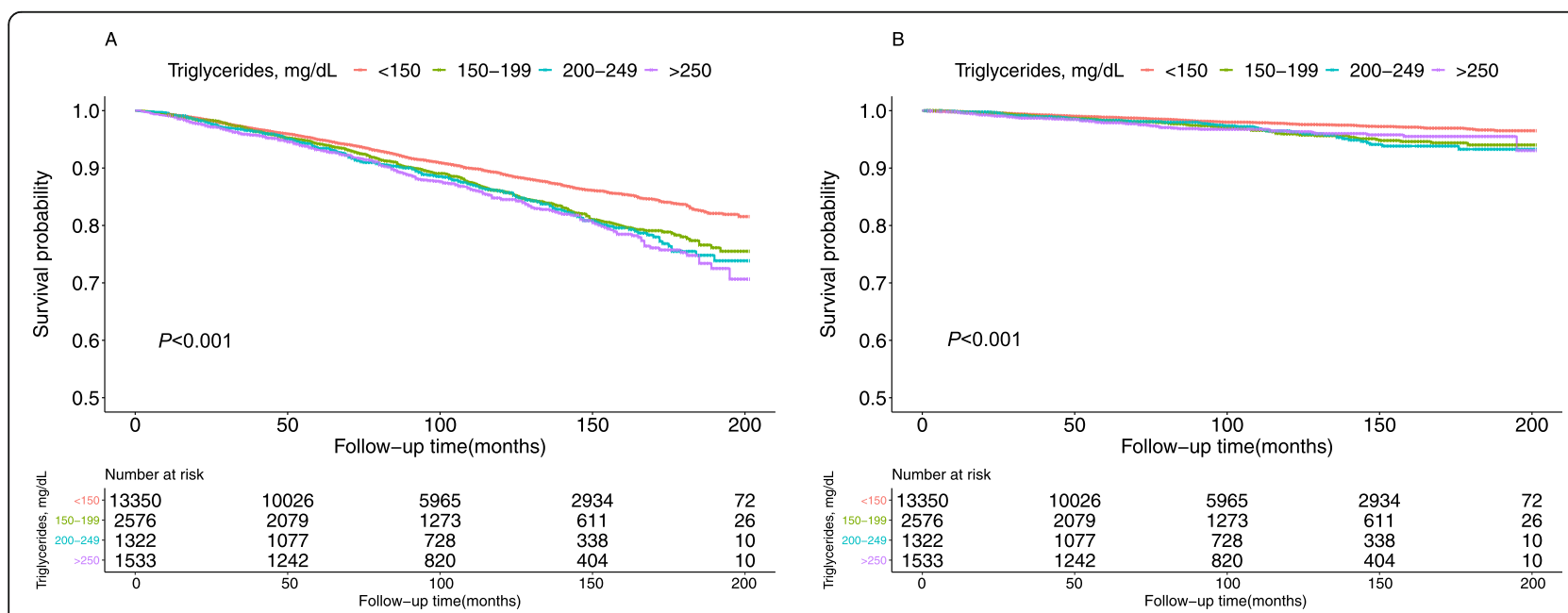

Fig. 2 Kaplan-Meier survival curves for all-cause (a) and cardiovascular (b) mortality 
Table 2 Multivariate Cox regression analysis of triglyceride levels with mortality in different models

\begin{tabular}{|c|c|c|c|}
\hline & $\begin{array}{l}\text { Model I } \\
\text { HR }(95 \% \mathrm{Cl}), P \text {-value }\end{array}$ & $\begin{array}{l}\text { Model II } \\
\text { HR }(95 \% \mathrm{Cl}), P \text {-value }\end{array}$ & $\begin{array}{l}\text { Model III } \\
\text { HR }(95 \% \mathrm{Cl}), P \text {-value }\end{array}$ \\
\hline \multicolumn{4}{|l|}{ All-cause mortality } \\
\hline Triglycerides (per mmol/L increment) & $1.17(1.13,1.21)<0.0001$ & $1.07(1.02,1.12) 0.0021$ & $1.08(1.02,1.15) 0.0085$ \\
\hline \multicolumn{4}{|l|}{ Triglycerides groups, mg/dL } \\
\hline$<150$ & 1.0 & 1.0 & 1.0 \\
\hline $150-200$ & $1.31(1.16,1.48)<0.0001$ & $1.00(0.89,1.13) 0.9414$ & $0.97(0.84,1.12) 0.7249$ \\
\hline $200-249$ & $1.39(1.19,1.62)<0.0001$ & $1.03(0.88,1.20) 0.7255$ & $1.06(0.89,1.27) 0.5172$ \\
\hline$\geq 250$ & $1.48(1.28,1.70)<0.0001$ & $1.32(1.14,1.51) 0.0001$ & $1.34(1.12,1.60) 0.0016$ \\
\hline$P$ for trend & $<0.001$ & 0.002 & 0.007 \\
\hline \multicolumn{4}{|l|}{ Cardiovascular mortality } \\
\hline Triglycerides (per mmol/L increment) & $1.22(1.13,1.31)<0.0001$ & $1.16(1.06,1.27) 0.0009$ & $1.10(0.97,1.24) 0.1482$ \\
\hline \multicolumn{4}{|l|}{ Triglycerides groups, mg/dL } \\
\hline$<150$ & 1.0 & 1.0 & 1.0 \\
\hline $150-200$ & $1.67(1.30,2.14)<0.0001$ & $1.28(1.00,1.65) 0.0504$ & $1.01(0.75,1.37) 0.9349$ \\
\hline $200-249$ & $1.77(1.29,2.41) 0.0004$ & $1.31(0.96,1.79) 0.0909$ & $1.19(0.82,1.71) 0.3544$ \\
\hline$\geq 250$ & $1.61(1.18,2.18) 0.0025$ & $1.53(1.12,2.08) 0.0068$ & $1.26(0.85,1.88) 0.2517$ \\
\hline$P$ for trend & $<0.001$ & 0.002 & 0.203 \\
\hline
\end{tabular}

Notes: Multivariate Cox regression was performed to examine the association between triglyceride levels and mortality

Data are shown in HRs and $95 \% \mathrm{Cl}$

$H R$ hazard ratios; $\mathrm{Cl}$ confidence intervals

Model I adjust for none

Model II adjust for age, gender and BMI

Model III adjust for age, gender, race, education level, smoking, body mass index, systolic blood pressure, estimated glomerular filtration rate, energy, C-reactive protein, total cholesterol, high density lipoprotein cholesterol, hypertension, diabetes, and medicine using (antihypertensive drugs, hypoglycemic agents, lipidlowering drugs, and antiplatelet drugs)

\section{Results}

\section{Baseline characteristics}

The present study included 18,781 participants (9130 males, mean age was 45.64 years). During the average follow-up period of 8.25 years, 1992 (10.61\%) allcause and $421(2.24 \%)$ cardiovascular death occurred. Table 1 has summarized the characteristics of participants at baseline. There were significant subgroup differences for all baseline variables according to TG levels. The differences in survival rate of all-cause (Fig. 2a) and cardiovascular (Fig. 2b) mortality according to the levels of TG were demonstrated in Fig. 2.

\section{The relationship between triglyceride and mortality}

As revealed in Table 2, for every $1 \mathrm{mmol} / \mathrm{L}$ increment in TG, TG (Model $3 \mathrm{HR}=1.08$, 95\%CI: 1.02, 1.15; $P=$ $0.0085)$ was significantly associated with all-cause mortality, but not for cardiovascular mortality (Model $3 \mathrm{HR}=$ 1.10, 95\%CI: 0.97, 1.24; $P=0.1482$ ). When using TG $<150$ $\mathrm{mg} / \mathrm{dL}$ as referent, the HRs for all-cause death were 0.97 $(0.84,1.12), 1.06(0.89,1.27)$ and $1.34(1.12,1.60)$ for TG

Table 3 The results of two-piecewise linear regression model between triglyceride and mortality

\begin{tabular}{|c|c|c|}
\hline & $\begin{array}{l}\text { All-cause mortality } \\
\text { HR }(95 \% \mathrm{Cl}) P \text {-value }\end{array}$ & $\begin{array}{l}\text { Cardiovascular disease mortality } \\
\text { HR }(95 \% \mathrm{Cl}) P \text {-value }\end{array}$ \\
\hline Cutoff value, mmol/L & $1.52 \mathrm{mmol} / \mathrm{L}(135 \mathrm{mg} / \mathrm{dL})$ & $1.10 \mathrm{mmol} / \mathrm{L}(97 \mathrm{mg} / \mathrm{dL})$ \\
\hline$<$ Cut-off value & $0.87(0.71,1.06) 0.1656$ & $0.59(0.25,1.39) 0.2247$ \\
\hline$\geq$ Cut-off value & $1.12(1.05,1.19) 0.0006$ & $1.12(0.99,1.27) 0.0747$ \\
\hline P for log likelihood ratio test & 0.026 & 0.159 \\
\hline
\end{tabular}

Notes: Multivariate linear regression was performed to examine the association between triglyceride levels and mortality

Data are shown in $\mathrm{HRs}$ and $95 \% \mathrm{Cl}$

$H R$ hazard ratios; $\mathrm{Cl}$ confidence intervals

Effect: age, gender, race, education level, smoking, body mass index, systolic blood pressure, estimated glomerular filtration rate, energy, C-reactive protein, total cholesterol, high density lipoprotein cholesterol, hypertension, diabetes, and medicine using (antihypertensive drugs, hypoglycemic agents, lipid-lowering drugs, and antiplatelet drugs) 
levels at 150-200, 200-249 and $\geq 250 \mathrm{mg} / \mathrm{dL}$ in Model 3, respectively. ( $P$ for trend $=0.0070$ ). Similarly, the HRs for death due to CVD were $1.01(0.75,1.37), 1.19(0.82,1.71)$ and $1.26(0.85,1.88)$ ( $P$ for trend $=0.2030)$, respectively.

As demonstrated in Table 3, the cut-off values of TG to predict death due to all-cause and cardiovascular diseases were $1.52 \mathrm{mmol} / \mathrm{L}(135 \mathrm{mg} / \mathrm{dL})$ and 1.10 $\mathrm{mmol} / \mathrm{L}(97 \mathrm{mg} / \mathrm{dL})$, respectively. Below the cut-off value, the HRs for of all-cause and CVD death were 0.87 (95\%CI: $0.71,1.06)$ and 0.59 (95\%CI: $0.25,1.39$ ) for every $1 \mathrm{mmol} / \mathrm{L}$ elevation in the level of serum TG. Above the cut-off value of TG levels, the HRs for of all-cause and CVD death were 1.12 (95\%CI: 1.05, 1.19) and 1.12 (95\%CI: $0.99,1.27)$ for every $1 \mathrm{mmol} / \mathrm{L}$ elevation in the level of serum TG, respectively. The risk of death due to all-cause was the lowest when TG was approximately $135 \mathrm{mg} / \mathrm{dL}$. Results from smoothing spline plots suggested that TG was linked with allcause death in dose-independent manner (Fig. 3a), but it was close to a linear relationship with cardiovascular mortality (Fig. 3b).

\section{Subgroup analysis}

Table 4 has summarized the results of subgroup analysis. When TG $\geq 135 \mathrm{mg} / \mathrm{dL}$, TG independently associated with all-cause death in subjects aged $\geq 65$ years (HR: $1.09,95 \% \mathrm{CI}$ : $0.99,1.19$ ), males (HR: 1.14, 95\%CI: 1.05, 1.23), people with $\mathrm{BMI} \geq 25 \mathrm{~kg} / \mathrm{m}^{2}$ (HR: 1.11, 95\%CI: 1.03, 1.19), White population (HR: 1.16, 95\%CI: 1.07, 1.27), people without taking lipid-lowering drugs (HR: 1.12, 95\%CI: 1.05, 1.20), people without diabetes (HR: 1.11, 95\%CI: 1.03, 1.19), people with hypertension (HR: 1.14, 95\%CI: 1.06, 1.22), people with diabetes (HR: 1.11, 95\%CI: 1.01, 1.22), and people with CVD
(HR: 1.11, 95\%CI: 1.03, 1.19). However, when TG was $\geq 97$ $\mathrm{mg} / \mathrm{dL}$, TG associated with cardiovascular mortality in subjects with $\mathrm{BMI}<25 \mathrm{~kg} / \mathrm{m}^{2}$ (HR: $1.44,95 \% \mathrm{CI}: 1.09,1.90$ ), males (HR: $1.21,95 \% \mathrm{CI}: 1.05,1.40$ ), people with hypertension (HR: 1.25, 95\%CI: 1.07, 1.46) and people with CVD (HR: 1.26, 95\%CI: 1.04, 1.52).

\section{Discussion}

In this study, results showed that elevated TG had independent association with all-cause mortality. When compared to people with $\mathrm{TG}<150 \mathrm{mg} / \mathrm{dL}, \mathrm{TG} \geq 250 \mathrm{mg} / \mathrm{dL}$ increased the risk of all-cause mortality increased by $34 \%$.

How serum TG related to all-cause mortality in the present study agreed with some previous studies $[2,6$, 7]. In this study, all-cause mortality risk was increased by $8 \%$ per $1 \mathrm{mmol} / \mathrm{L}$ TG increment. Meanwhile, the association between TG and CVD death did not reach significance level. A meta-analysis demonstrated that the risk of CVD death elevated by $13 \%(P<0.001)$ per 1 $\mathrm{mmol} / \mathrm{L}$ TG increment, suggesting TG associated with CVD mortality and was independent from multiple cardiovascular risk factors [7]. In addition, adjusted spline curves analyze showed when TG was greater than 135 $\mathrm{mg} / \mathrm{dL}$, the risk of all-cause mortality was elevated, suggesting a nonlinear relationship between TG and mortality. This finding was similar to previous studies $[7,17]$. When $\mathrm{TG}<135 \mathrm{mg} / \mathrm{dL}$, TG was inversely associated with all-cause mortality. However, there was a study found that TG ranged 100 to $149 \mathrm{mg} / \mathrm{dL}$ might also increase the risk for mortality [18]. The differences in the study population may be the main reasons for the discrepancy in findings.

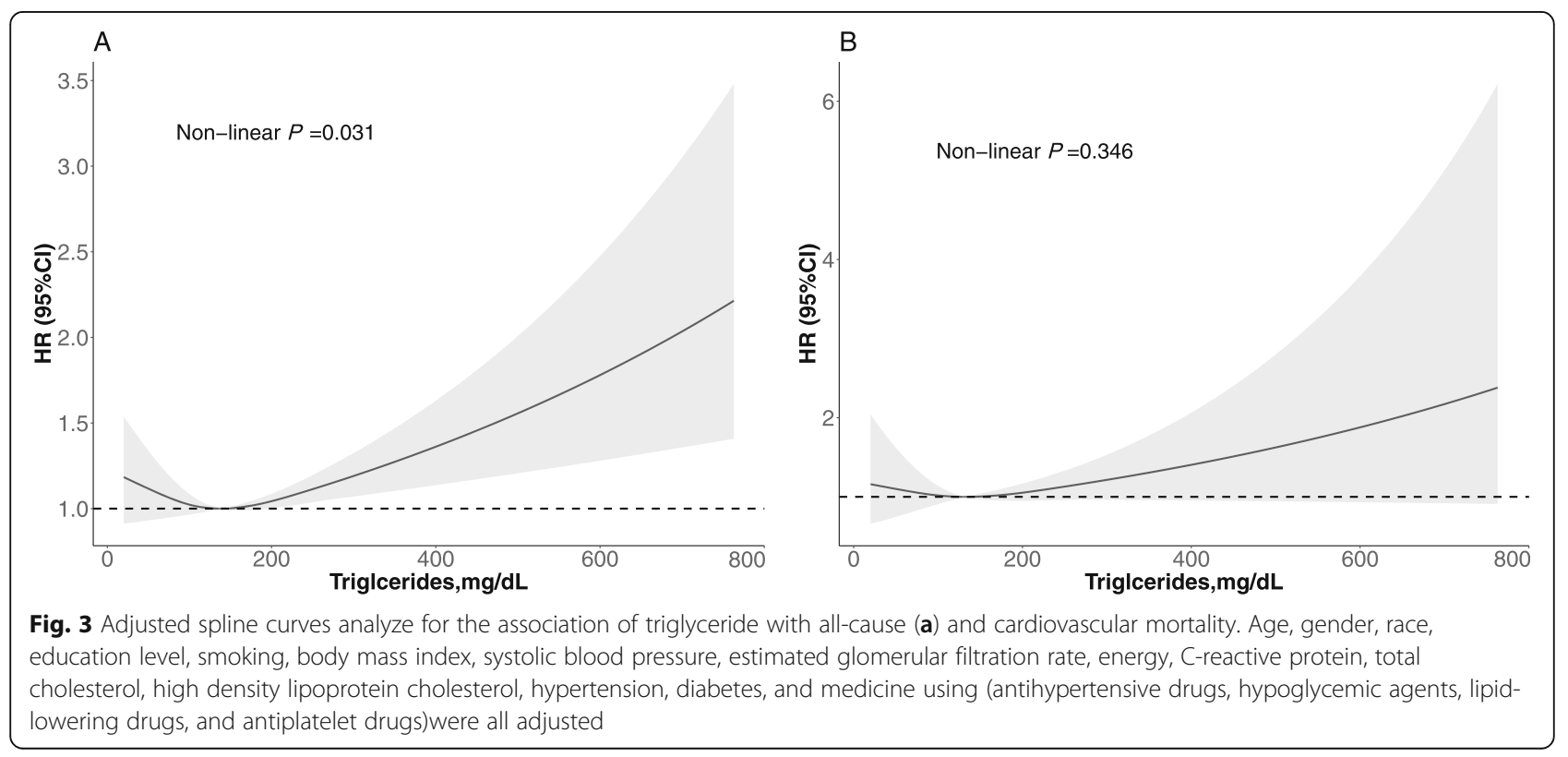


Table 4 Subgroup analysis of triglycerides levels with mortality

\begin{tabular}{|c|c|c|c|c|c|c|c|}
\hline \multirow[b]{2}{*}{$\begin{array}{l}\text { Cutoff value, } \\
\mathrm{mmol} / \mathrm{L}\end{array}$} & \multirow[t]{2}{*}{ Number } & \multicolumn{2}{|c|}{$\begin{array}{l}\text { All-cause mortality } \\
\text { HR }(95 \% \mathrm{CI}) P \text {-value }\end{array}$} & \multirow[t]{2}{*}{$\begin{array}{l}\mathrm{P} \text { for log likelihood } \\
\text { ratio test }\end{array}$} & \multicolumn{2}{|c|}{$\begin{array}{l}\text { Cardiovascular disease mortality } \\
\text { HR }(95 \% \mathrm{Cl}) P \text {-value }\end{array}$} & \multirow[t]{2}{*}{$\begin{array}{l}P \text { for log likelihood } \\
\text { ratio test }\end{array}$} \\
\hline & & $<1.52$ & $\geq 1.52$ & & $<1.10$ & $\geq 1.10$ & \\
\hline \multicolumn{8}{|l|}{ Age, years } \\
\hline$\geq 65$ & 2502 & $\begin{array}{l}0.78(0.60,1.01) \\
0.0558\end{array}$ & $\begin{array}{l}1.09(0.99,1.19) \\
0.0654\end{array}$ & 0.024 & $\begin{array}{l}0.54(0.19,1.51) \\
0.2400\end{array}$ & $\begin{array}{l}1.00(0.84,1.19) \\
0.9968\end{array}$ & 0.267 \\
\hline$<65$ & 9135 & $\begin{array}{l}1.21(0.87,1.68) \\
0.2640\end{array}$ & $\begin{array}{l}1.05(0.95,1.15) \\
0.3327\end{array}$ & 0.436 & $\begin{array}{l}1.49(0.30,7.35) \\
0.6273\end{array}$ & $\begin{array}{l}1.15(0.96,1.38) \\
0.1206\end{array}$ & 0.757 \\
\hline \multicolumn{8}{|l|}{ Gender } \\
\hline Male & 5659 & $\begin{array}{l}0.91(0.69,1.19) \\
0.4874\end{array}$ & $\begin{array}{l}1.14(1.05,1.23) \\
0.0016\end{array}$ & 0.136 & $\begin{array}{l}0.85(0.29,2.50) \\
0.7651\end{array}$ & $\begin{array}{l}1.21(1.05,1.40) \\
0.0096\end{array}$ & 0.527 \\
\hline Female & 5978 & $\begin{array}{l}0.89(0.65,1.22) \\
0.4596\end{array}$ & $\begin{array}{l}1.08(0.97,1.20) \\
0.1491\end{array}$ & 0.277 & $\begin{array}{l}0.44(0.10,2.00) \\
0.2873\end{array}$ & $\begin{array}{l}0.92(0.72,1.18) \\
0.5239\end{array}$ & 0.367 \\
\hline \multicolumn{8}{|c|}{ Body mass index, $\mathrm{kg} / \mathrm{m}^{2}$} \\
\hline$\geq 25$ & 8102 & $\begin{array}{l}0.90(0.70,1.16) \\
0.4312\end{array}$ & $\begin{array}{l}1.11(1.03,1.19) \\
0.0036\end{array}$ & 0.143 & $\begin{array}{l}0.70(0.22,2.19) \\
0.5437\end{array}$ & $\begin{array}{l}1.09(0.94,1.26) \\
0.2783\end{array}$ & 0.471 \\
\hline$<25$ & 3535 & $\begin{array}{l}0.78(0.54,1.12) \\
0.1821\end{array}$ & $\begin{array}{l}1.23(1.03,1.47) \\
0.0244\end{array}$ & 0.050 & $\begin{array}{l}0.43(0.11,1.67) \\
0.2241\end{array}$ & $\begin{array}{l}1.44(1.09,1.90) \\
0.0095\end{array}$ & 0.105 \\
\hline \multicolumn{8}{|l|}{ Race } \\
\hline Non-white & 6114 & $\begin{array}{l}1.00(0.74,1.35) \\
0.9952\end{array}$ & $\begin{array}{l}1.05(0.96,1.16) \\
0.2861\end{array}$ & 0.757 & $\begin{array}{l}0.34(0.10,1.11) \\
0.0738\end{array}$ & $\begin{array}{l}1.17(0.98,1.39) \\
0.0916\end{array}$ & 0.054 \\
\hline White & 5523 & $\begin{array}{l}0.79(0.60,1.05) \\
0.1088\end{array}$ & $\begin{array}{l}1.16(1.07,1.27) \\
0.0007\end{array}$ & 0.016 & $\begin{array}{l}1.08(0.30,3.96) \\
0.9055\end{array}$ & $\begin{array}{l}1.07(0.89,1.27) \\
0.4852\end{array}$ & 0.982 \\
\hline \multicolumn{8}{|c|}{ Lipid-lowering drugs } \\
\hline No & 10,251 & $\begin{array}{l}0.91(0.73,1.15) \\
0.4476\end{array}$ & $\begin{array}{l}1.12(1.05,1.20) \\
0.0015\end{array}$ & 0.114 & $\begin{array}{l}0.53(0.20,1.42) \\
0.2078\end{array}$ & $\begin{array}{l}1.14(0.98,1.32) \\
0.0813\end{array}$ & 0.147 \\
\hline Yes & 1386 & $\begin{array}{l}0.75(0.48,1.17) \\
0.2035\end{array}$ & $\begin{array}{l}1.11(0.96,1.29) \\
0.1696\end{array}$ & 0.126 & $\begin{array}{l}1.05(0.16,6.85) \\
0.9565\end{array}$ & $\begin{array}{l}1.07(0.83,1.37) \\
0.6183\end{array}$ & 0.990 \\
\hline \multicolumn{8}{|l|}{ Hypertension } \\
\hline No & 6779 & $\begin{array}{l}0.87(0.59,1.29) \\
0.4962\end{array}$ & $\begin{array}{l}1.07(0.94,1.23) \\
0.2792\end{array}$ & 0.350 & $\begin{array}{l}2.16(0.22,21.49) \\
0.5106\end{array}$ & $\begin{array}{l}0.95(0.68,1.34) \\
0.7752\end{array}$ & 0.483 \\
\hline Yes & 4858 & $\begin{array}{l}0.81(0.64,1.03) \\
0.0903\end{array}$ & $\begin{array}{l}1.14(1.06,1.22) \\
0.0006\end{array}$ & 0.013 & $\begin{array}{l}0.46(0.18,1.17) \\
0.1035\end{array}$ & $\begin{array}{l}1.16(1.01,1.33) \\
0.0358\end{array}$ & 0.066 \\
\hline \multicolumn{8}{|l|}{ Diabetes } \\
\hline No & 9867 & $\begin{array}{l}0.90(0.71,1.14) \\
0.3882\end{array}$ & $\begin{array}{l}1.14(1.04,1.25) \\
0.0065\end{array}$ & 0.101 & $\begin{array}{l}0.85(0.30,2.43) \\
0.7648\end{array}$ & $\begin{array}{l}0.91(0.72,1.14) \\
0.4063\end{array}$ & 0.911 \\
\hline Yes & 1770 & $\begin{array}{l}0.82(0.55,1.22) \\
0.3276\end{array}$ & $\begin{array}{l}1.11(1.01,1.22) \\
0.0287\end{array}$ & 0.162 & $\begin{array}{l}0.43(0.09,2.17) \\
0.3091\end{array}$ & $\begin{array}{l}1.25(1.07,1.46) \\
0.0054\end{array}$ & 0.223 \\
\hline \multicolumn{8}{|l|}{ CVD } \\
\hline No & 10,646 & $\begin{array}{l}0.93(0.74,1.18) \\
0.5569\end{array}$ & $\begin{array}{l}1.10(1.02,1.18) \\
0.0142\end{array}$ & 0.217 & $\begin{array}{l}0.83(0.28,2.43) \\
0.7333\end{array}$ & $\begin{array}{l}1.01(0.85,1.20) \\
0.8977\end{array}$ & 0.727 \\
\hline Yes & 991 & $\begin{array}{l}0.64(0.42,0.98) \\
0.0405\end{array}$ & $\begin{array}{l}1.18(1.04,1.34) \\
0.0112\end{array}$ & 0.012 & $\begin{array}{l}0.35(0.07,1.64) \\
0.1826\end{array}$ & $\begin{array}{l}1.26(1.04,1.52) \\
0.0192\end{array}$ & 0.123 \\
\hline
\end{tabular}

Notes: Multivariate Cox regression was performed to examine the association between triglyceride levels and mortality

Data are shown in $\mathrm{HRs}$ and $95 \% \mathrm{Cl}$

$H R$ hazard ratios; $C l$ confidence intervals; $C A D$ coronary heart disease

When analyzing a subgroup variable, age, gender, race, education level, smoking, body mass index, systolic blood pressure, estimated glomerular filtration rate, Creactive protein, energy, total cholesterol, high density lipoprotein cholesterol, hypertension, diabetes, medicine use (antihypertensive drugs, hypoglycemic agents, lipid-lowering drugs, and antiplatelet drugs) were all adjusted except the variable itself

In the present study, the stratified analysis indicated no significant relationship between TG and cardiovascular mortality in all subgroups. However, the relationship was differed by age, gender, race, BMI, comorbidities, and the use of lipid-lowering drugs. Female participants have a lower risk of death than males, and could be explained by the role of estrogen. Previous research suggested that TG levels were significantly influenced by 
the endogenous hormonal environment [19-21]. It was also found that the relationship between TG and death was stronger among Whites, probably due to the difference in eating habits and genetic variations between ethnic groups.

The specific mechanism by which TG increased the risk of death has not been fully elucidated. First, animal and human experiments indicated that excessive TG levels were often accompanied by higher inflammation or oxidative stress $[22,23]$. Second, circulating TG could passed the blood-brain and induce insulin receptor resistance [24]. Third, genetic variation of lipoprotein lipase, apolipoprotein C3 and lipase maturation factor-1 may play an important role [25]. Apolipoprotein C-III could associate with hypertriglyceridemia and CVD [26]. Although there were still many uncertainties in blood lipid metabolism, proprotein convertase subtilisin/kexin type 9 (PCSK9) has demonstrated an important role in lipid metabolism [27-29]. Moreover, high intrahepatic or circulating PCSK9 levels could increase TG storage and secretion, thus leading to a higher risk of CVD. These observations suggested the use of PCSK9 inhibitors to prevent CVD [30].

\section{Study strength and limitations}

The strength of this study was to link with national data, which helped us to elucidate prospective relationship between TG and mortality. However, this study has some limitations. First, some covariates were self-reported. Second, there were other confounders not being adjusted, such as exercise and cardiovascular risk scores. Third, data on serum lipid was only collected once at baseline, and it was unclear how the changes in TG over time might influence the association with mortality. In addition, sample size was reduced due to incomplete data collection of serum lipids in NHANES. Finally, the study findings were mainly applicable to the American population and cannot be extrapolated to other countries.

\section{Conclusion}

Elevated TG was independently associated with all-cause mortality, but no significant relationship with cardiovascular death. The results might also suggest non-linear correlation between TG and all-cause death. More attention should be paid to the association of TG with CVDrelated mortality, and the management and monitoring of TG should be strengthened. In addition, the relationship between TG and cause-specific deaths is still unclear. More basic and clinical researches are still needed to clarify this relationship.

\section{Abbreviations}

CDC: Centers for Disease Control and Prevention; eGFR: Estimated glomerular filtration rate; CVD: Cardiovascular diseases; NHANES: National Health and Nutrition Examination Surveys; HR: Hazard ratio; Cl: Confidence interval;
TG: Triglyceride; LDL-C: Low density lipoprotein cholesterol; HDL-C: High density lipoprotein cholesterol; TC: Total cholesterol; SBP: Systolic blood pressure; DBP: Diastolic blood pressure; BMI: Body mass index; HbA1C: Hemoglobin A1C

\section{Acknowledgements \\ None.}

\section{Authors' contributions}

Yu-qing HUANG, Ying-qing FENG and Bin ZHANG contributed to study design. Yu-qing HUANG, Xiao-cong LIU and Kenneth Lo contributed to data analysis and manuscript drafting. Yu-qing HUANG, Xiao-cong LIU, Kenneth Lo contributed to data downloading. All authors contributed to manuscript revising and have approved the final article.

\section{Funding}

This work was supported by the Science and Technology Plan Program of Guangzhou (No. 201803040012), the Key Area R\&D Program of Guangdong Province (No. 2019B020227005) and the National Key Research and Development Program of China (No.2017YFC1307603).

\section{Availability of data and materials}

Data are from the NHANES Study. Data are available in a public, open access repository

\section{Ethics approval and consent to participate}

The survey protocol was approved by the Institutional Review Board of the Centers for Disease Control and Prevention (Protocol 98-12, 2005-06 and 2011-17). All patients received informed consent before the start of the study.

Consent for publication

All authors approved and agreed to publish the final version of the manuscript.

\section{Competing interests}

The authors declare that they have no conflict of interest.

\section{Author details}

'Department of Cardiology, Guangdong Cardiovascular Institute, Guangdong Provincial People's Hospital, Guangdong Academy of Medical Sciences, South China University of Technology School of Medicine, No. 106, Zhongshan Second Road, Yuexiu District, Guangzhou 510080, China. ${ }^{2}$ Centre for Global Cardiometabolic Health, Department of Epidemiology, Brown University, Providence, RI, USA. ${ }^{3}$ Department of Applied Biology and Chemical Technology, The Hong Kong Polytechnic University, Hung Hom, Hong Kong, China.

Received: 31 July 2020 Accepted: 6 October 2020

Published online: 15 October 2020

\section{References}

1. Sarwar N, Danesh J, Eiriksdottir G, Sigurdsson G, Wareham N, Bingham S, et al. Triglycerides and the risk of coronary heart disease: 10,158 incident cases among 262,525 participants in 29 Western prospective studies. Circulation. 2007;115(4):450-8.

2. Kasai T, Miyauchi K, Yanagisawa N, Kajimoto K, Kubota N, Ogita M, et al. Mortality risk of triglyceride levels in patients with coronary artery disease. Heart. 2013:99(1):22-9.

3. Toth PP, Philip S, Hull M, Granowitz C. Elevated triglycerides ( $>/=150 \mathrm{mg} / \mathrm{dL}$ ) and high triglycerides (200-499 mg/dL) are significant predictors of new heart failure diagnosis: a real-world analysis of high-risk statin-treated patients. Vasc Health Risk Manag. 2019;15:533-8.

4. Toth PP, Philip S, Hull M, Granowitz C. Association of Elevated Triglycerides with Increased Cardiovascular Risk and Direct Costs in statin-treated patients. Mayo Clin Proc. 2019:94(9):1670-80.

5. Patel A, Barzi F, Jamrozik K, Lam TH, Ueshima H, Whitlock G, et al. Serum triglycerides as a risk factor for cardiovascular diseases in the Asia-Pacific region. Circulation. 2004;110(17):2678-86.

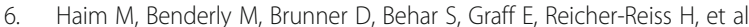
Elevated serum triglyceride levels and long-term mortality in patients with 
coronary heart disease: the Bezafibrate infarction prevention (BIP) registry. Circulation. 1999;100(5):475-82.

7. Liu J, Zeng FF, Liu ZM, Zhang CX, Ling WH, Chen YM. Effects of blood triglycerides on cardiovascular and all-cause mortality: a systematic review and meta-analysis of 61 prospective studies. Lipids Health Dis. 2013;12:159.

8. Marston NA, Giugliano RP, Im K, Silverman MG, O'Donoghue ML, Wiviott SD, et al. Association between triglyceride lowering and reduction of cardiovascular risk across multiple lipid-lowering therapeutic classes: a systematic review and meta-regression analysis of randomized controlled trials. Circulation. 2019:140(16):1308-17.

9. Criqui MH, Heiss G, Cohn R, Cowan LD, Suchindran CM, Bangdiwala S, et al. Plasma triglyceride level and mortality from coronary heart disease. N Engl J Med. 1993;328(17):1220-5.

10. Khawaja OA, Hatahet H, Cavalcante J, Khanal S, Al-Mallah MH. Low admission triglyceride and mortality in acute coronary syndrome patients. Cardiol J. 2011;18(3):297-303

11. Bucholz EM, Rodday AM, Kolor K, Khoury MJ, de Ferranti SD. Prevalence and predictors of cholesterol screening, awareness, and statin treatment among US adults with familial hypercholesterolemia or other forms of severe dyslipidemia (1999-2014). Circulation. 2018;137(21):2218-30.

12. Doran B, Guo Y, Xu J, Weintraub H, Mora S, Maron DJ, et al. Prognostic value of fasting versus nonfasting low-density lipoprotein cholesterol levels on long-term mortality: insight from the National Health and nutrition examination survey III (NHANES-III). Circulation. 2014;130(7):546-53.

13. Friedewald WT, Levy RI, Fredrickson DS. Estimation of the concentration of low-density lipoprotein cholesterol in plasma, without use of the preparative ultracentrifuge. Clin Chem. 1972;18(6):499-502.

14. James PA, Oparil S, Carter BL, Cushman WC, Dennison-Himmelfarb C, Handler J, et al. 2014 evidence-based guideline for the management of high blood pressure in adults: report from the panel members appointed to the eighth joint National Committee (JNC 8). JAMA. 2014;311(5):507-20.

15. Association AD. Classification and diagnosis of diabetes: standards of medical Care in Diabetes-2018. Diabetes Care. 2018;41(Suppl 1):S13-27.

16. Huang YQ, Liu L, Huang JY, Lo K, Chen CL, Yu YL, et al. Prehypertension and risk for all-cause and cardiovascular mortality by diabetes status: results from the national health and nutrition examination surveys. Ann Transl Med. 2020;8(6):323.

17. Pikhart H, Hubacek JA, Peasey A, Kubinova R, Bobak M. Association between fasting plasma triglycerides, all-cause and cardiovascular mortality in Czech population. Results from the HAPIEE study. Physiol Res. 2015;64(Suppl 3): S355-61.

18. Klempfner R, Erez A, Sagit BZ, Goldenberg I, Fisman E, Kopel E, et al. Elevated triglyceride level is independently associated with increased allcause mortality in patients with established coronary heart disease: twentytwo-year follow-up of the Bezafibrate infarction prevention study and registry. Circ Cardiovasc Qual Outcomes. 2016;9(2):100-8.

19. Jaworski K, Sarkadi-Nagy E, Duncan RE, Ahmadian M, Sul HS. Regulation of triglyceride metabolism. IV. Hormonal regulation of lipolysis in adipose tissue. Am J Physiol Gastrointest Liver Physiol. 2007;293(1):G1-4

20. Barnett JB, Woods MN, Lamon-Fava S, Schaefer EJ, McNamara JR, Spiegelman D, et al. Plasma lipid and lipoprotein levels during the follicular and luteal phases of the menstrual cycle. J Clin Endocrinol Metab. 2004; 89(2):776-82.

21. Vashishta S, Gahlot S, Goyal R. Effect of menstrual cycle phases on plasma lipid and lipoprotein levels in regularly menstruating women. J Clin Diagn Res. 2017;11(5):C5-7.

22. Al-Aubaidy HA, Jelinek HF. Oxidative stress and triglycerides as predictors of subclinical atherosclerosis in prediabetes. Redox Rep. 2014;19(2):87-91.

23. Brito A, Lima GM, Farias LM, Rodrigues L, Carvalho V, Pereira C, et al. Lycopene-Rich Extract from Red Guava (Psidium guajava L.) Decreases Plasma Triglycerides and Improves Oxidative Stress Biomarkers on Experimentally-Induced Dyslipidemia in Hamsters. Nutrients. 2019;11(2):393.

24. Banks WA, Farr SA, Salameh TS, Niehoff ML, Rhea EM, Morley JE, et al. Triglycerides cross the blood-brain barrier and induce central leptin and insulin receptor resistance. Int J Obes. 2018;42(3):391-7.

25. Tada H, Kawashiri MA. Genetic variations, triglycerides, and atherosclerotic disease. J Atheroscler Thromb. 2019;26(2):128-31.

26. Luo M, Peng D. The emerging role of apolipoprotein C-III: beyond effects on triglyceride metabolism. Lipids Health Dis. 2016;15(1):184.

27. Cesaro A, Bianconi V, Gragnano F, Moscarella E, Fimiani F, Monda E, et al. Beyond cholesterol metabolism: the pleiotropic effects of proprotein convertase subtilisin/kexin type 9 (PCSK9). Genetics, mutations, expression, and perspective for long-term inhibition. Biofactors. 2020;46(3):367-80.

28. Lagace TA. PCSK9 and LDLR degradation: regulatory mechanisms in circulation and in cells. Curr Opin Lipidol. 2014;25(5):387-93.

29. Demers A, Samami S, Lauzier B, Des Rosiers C, Ngo SE, Ong H, et al. PCSK9 induces CD36 degradation and affects long-chain fatty acid uptake and triglyceride metabolism in adipocytes and in mouse liver. Arterioscler Thromb Vasc Biol. 2015;35(12):2517-25.

30. Theocharidou E, Papademetriou M, Reklou A, Sachinidis A, Boutari C, Giouleme O. The role of PCSK9 in the pathogenesis of non-alcoholic fatty liver disease and the effect of PCSK9 inhibitors. Curr Pharm Des. 2018;24(31): $3654-7$.

\section{Publisher's Note}

Springer Nature remains neutral with regard to jurisdictional claims in published maps and institutional affiliations.
Ready to submit your research? Choose BMC and benefit from:

- fast, convenient online submission

- thorough peer review by experienced researchers in your field

- rapid publication on acceptance

- support for research data, including large and complex data types

- gold Open Access which fosters wider collaboration and increased citations

- maximum visibility for your research: over $100 \mathrm{M}$ website views per year

At BMC, research is always in progress.

Learn more biomedcentral.com/submissions 